\title{
Lichen Biodiversity and Conservation Status in the Copeland Forest Resources Management Area: A Lichen-Rich Second-Growth Forest in Southern Ontario
}

\author{
R. Troy McMullin ${ }^{1, *}$ and James C. Lendemer ${ }^{2}$
}

${ }^{1}$ Biodiversity Institute of Ontario Herbarium, Department of Integrative Biology, University of Guelph, Guelph, Ontario N1G 2W1 Canada

${ }^{2}$ Institute of Systematic Botany, New York Botanical Garden, Bronx, New York 10458-5126 USA

${ }^{*}$ Corresponding author; email: rmcmulli@uoguelph.ca

McMullin, R. Troy, and James C. Lendemer. 2013. Lichen biodiversity and conservation status in the Copeland Forest Resources Management Area: a lichen-rich second-growth forest in southern Ontario. Canadian Field-Naturalist 127(3): 240254.

Southern Ontario is the most densely populated region in Canada. As a result, ubanization, industrialization, and agriculture are extensive. Few ecosystems in the region have been unaltered, and second-growth forests now dominate the remaining natural landscape. To better understand the lichen diversity in these second-growth forests, we inventoried 24 distinct vegetation communites in the Copeland Forest Resources Management Area (1780 ha) located between Barrie and Orillia in September and October 2011, recording 154 species in 79 genera. One species, Lecidea sarcogynoides, was collected for the first time in Canada and is reported for the first time in North America; one additional species, Micarea micrococca, was collected for the first time in Ontario and is reported for the first time in Canada; three species that have previously been collected in Ontario-Bellemerea cinereorufescens, Phlyctis speirea, and Xanthoparmelia angustiphylla - are reported for the first time in the province; and Candelariella lutella was collected and is reported for the second time in Ontario and the third time in Canada. In addition, six species with a provincial status rank of S1 (critically imperilled) or S2 (imperilled) were located: Arthonia byssacea, Arthonia ruana, Chaenothecopsis pusiola, Cresponea chloroconia, Pachyphiale fagicola, and Placynthiella uliginosa. Our results show that second-growth forests can be important refugia for lichen diversity. The majority of the lichen diversity within the Copeland Forest was contained in a small number of sites (6 of 24). This suggests that management strategies should integrate lichen diversity by targeting species-rich areas. We found that sites with a high variation in: canopy closure, tree species, tree age, moisture, and the presence of snags had the highest lichen diversity. Forest managers in southern Ontario can use our results to identify species-rich areas on their properties.

Key Words: Lecidea sarcogynoides; Bellemerea cinereorufescens; Phlyctis speirea; Xanthoparmelia angustiphylla; Candelariella lutella; Arthonia byssacea; Arthonia ruana; Chaenothecopsis pusiola; Cresponea chloroconia; Pachyphiale fagicola; Placynthiella uliginosa; sustainable forest management; biodiversity; biogeography; Appalachian-Great Lakes; Copeland Forest Resources Management Area; Ontario

\section{Introduction}

The lichen biota of Ontario is rich and abundant; approximately 1070 species are currently known from the province (Newmaster et al., in press), and the lichen biomass in some areas is over $9000 \mathrm{~kg} / \mathrm{ha} \mathrm{(McMullin}$ et al. 2011). The total terrestrial area of Ontario is $917741 \mathrm{~km}^{2}$, which covers a number of diverse ecoregions (Perera et al. 2001). Many species have specialized habitat requirements, and the diversity of the landscape accounts for the relatively large number of lichens known from the province.

Most lichen species require specific habitats, microhabitats, and substrates (Schmitt and Slack 1990; Kuusinen 1996; McMullin et al. 2008). This specificity includes lichen communities that change in composition along a broad bioclimatic gradient from the Arctic/ alpine zone through boreal, temperate, and tropical zones. In Ontario, all but tropical conditions are present (Ahti 1964; Gowan and Brodo 1988; Brodo et al. 2001).

Specific ecosystems, such as bogs, cliffs, coastal areas, deserts, forests, prairies, and swamps, are the next level of division that shapes lichen community structure (Nash 2008; Brodo et al. 2001). Much more specifically, many lichens require particular substrates. The substrate requirements for some species are broad, and they may grow on a range of types of bark, leaves, rocks, soil, or wood, while other lichen species require substrates as specific as particular tree species at a particular stage of development (Söderström 1988; Botting and Delong 2009; McMullin et al. 2010). Defined amounts of light and moisture are also of primary importance for many lichen species (Kenkel and Bradfield 1986; Coxson and Coyle 2003; Coxson and Stevenson 2007). An understanding of the habitats that are most important for lichen diversity is therefore required to manage these organisms effectively.

Managing lichen diversity in southern Ontario presents a number of challenges. The first is a fundamental lack of baseline data: comprehensive knowledge of the lichen biota before it was altered by air pollution and development is lacking. Many lichen species are intolerant of air pollution (Henderson 2000). Although point source emissions may be controlled, much of the 
air pollution in southern Ontario is wide-ranging and difficult to isolate. Extensive urban, industrial, and agricultural development is the cause of this air pollution (Bates and Sizto 1987), but this development has also resulted in the loss of much of the old-growth forest that once dominated the landscape (Henry and Quinby 2010).

Many lichen species require habitats that are characteristic of old-growth forests (Lesica et al. 1991; Goward 1994; McMullin et al. 2008), and most of those species are sensitive to changes in their environments (Esseen and Renhorn 1998; Chen et al. 1993; McMullin et al. 2010). An important part of managing lichen diversity in southern Ontario now means understanding how species colonize second-growth forests.

The purpose of this study was to better understand the lichen diversity of second-growth forests in southern Ontario by examining a representative sampling of habitats in the Copeland Forest Resources Management Area north of Barrie. Specific objectives were to (1) determine the number of lichen species in the Copeland, (2) determine the frequency of occurrence of each lichen species, (3) resolve whether alpha diversity differs among different sampling sites (vegetation communities), and (4) identify sites with high lichen richness.

These objectives aim to produce a baseline inventory which can be used to monitor any changes in lichen diversity over time. Identifying the sites that are most important for capturing lichen diversity will assist forest managers with producing management strategies that target these areas. The results can be used in the development of sustainable management strategies, both in the study area and throughout southern Ontario in other second-growth forests.

\section{Methods}

\section{Study area}

The Copeland Forest Resources Management Area is located in southern Ontario approximately $15 \mathrm{~km}$ north of Barrie and $15 \mathrm{~km}$ west of Orillia (Figure 1). It lies between $44^{\circ} 32^{\prime} 34^{\prime \prime}$ and $44^{\circ} 35^{\prime} 43^{\prime \prime} \mathrm{N}$ and between $79^{\circ} 44^{\prime} 31^{\prime \prime}$ and $79^{\circ} 39^{\prime} 19^{\prime \prime} W$. Highway 400 runs along the length of the northwest side of the Copeland, and Horseshoe Valley Road runs the length of the southeast side. A continuous property covering 1780 hectares (Golas 1980), the Copeland Forest is a multiuse natural recreation area that is used mainly by

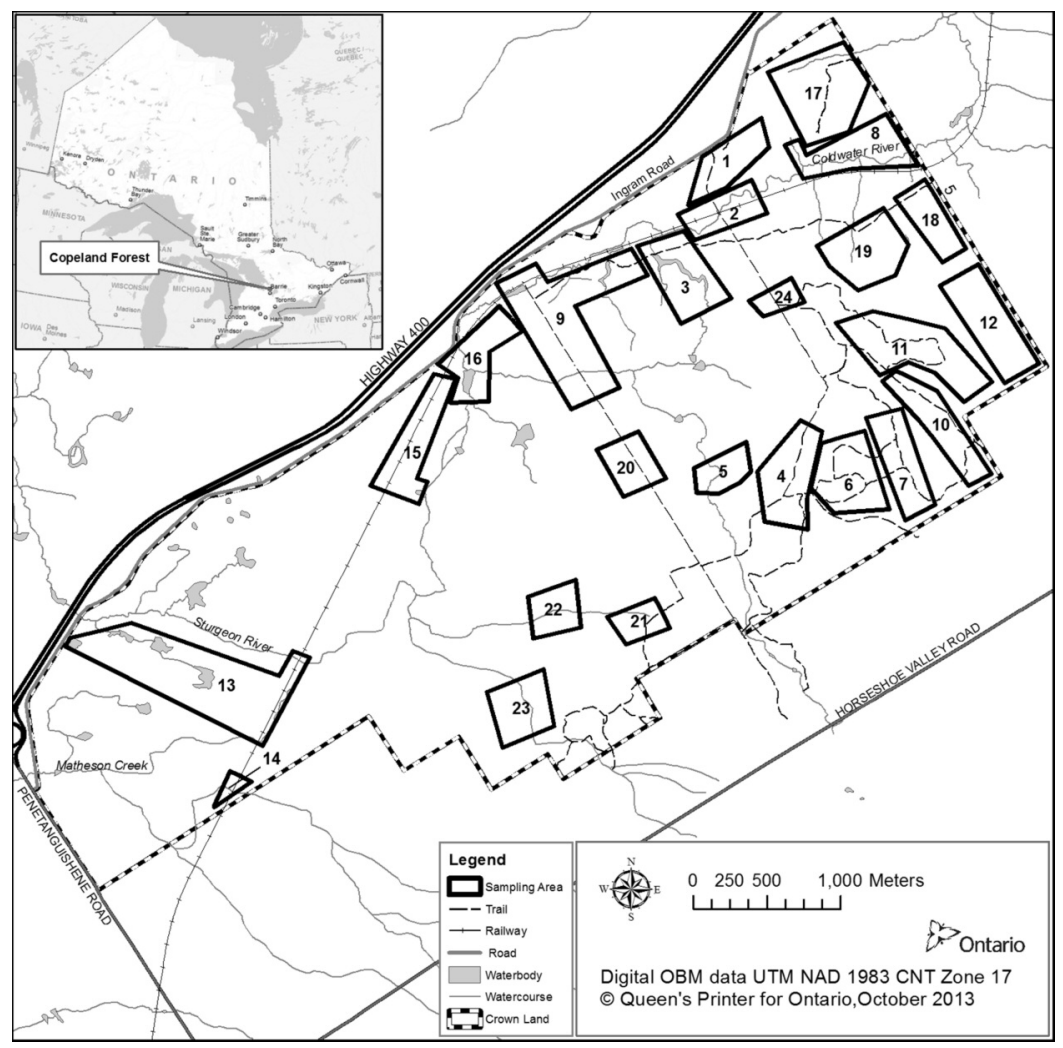

FIGURE 1. The Copeland Forest Resources Management Area with the 24 sampling sites illustrated. Sampling sites are described in Table 1. 
mountain bikers, horseback riders, hikers, hunters, and crosscountry skiers. An extensive network of trails in the Copeland Forest is maintained by Horseshoe Resort for its clientele, particularly for crosscountry skiing. One of the primary rail lines connecting southern Ontario to western Canada also runs through the forest, with multiple diesel-powered trains passing through daily.

The study area lies within the Great Lakes-St. Lawrence Forest Region and is characterized by rolling hills, treed wetlands, small ponds, and deciduous mixedwood and coniferous forests (Rowe 1972). The mean regional temperature in January is $-8.4^{\circ} \mathrm{C}$ and in July it is $20.6^{\circ} \mathrm{C}$ (Environment Canada 2012). The average annual rainfall is $750.6 \mathrm{~mm}$ and the average snowfall is $292.6 \mathrm{~cm}$ (Environment Canada 2012).

Virtually all of the forested land in the study area is second-growth. The first recorded evidence of harvesting dates from 1872, but unrecorded harvesting may have occured before that date. Harvesting continued until a mill fire on May 7, 1975 (Golas 1980). Assisted regeneration of the forest began in the 1930s and continued until the 1970s. During this time, 800,000 nursery seedlings, primarily conifers were planted (Golas 1980).

\section{Lichen diversity}

For the purposes of this study, lichen diversity is defined as lichen richness (number of species) plus abundance (frequency of occurrence of a species). To assess lichen richness, we established 24 sites that covered all of the major vegetation communities (Figure 1 and Table 1). Vegetation communities were identified using the Ontario Ministry of Natural Resources (OMNR) vegetation map for the Copeland Forest Resources Management Area. Many vegetation communities were represented by two or more spatially separate occurrences in the study area, and at least one vegetation community was included in each of the 24 sampling sites established. Once sampling sites had been selected, they were ground-truthed to determine vegetation communities accurately. Sampling sites varied in size and shape, as they followed the dominant vegetation communities.

All substrates throughout the total area of each site were examined for lichen species on September 15-18, September 30, and October 1-4, 2011. Our inventory methods follow those of Newmaster et al. (2005), who showed that examining large areas (referred to as floristic habitat sampling) captures cryptogam diversity more effectively than establishing smaller representative plots. Using floristic habitat sampling, our dominant mesohabitats were each of the 24 sampling sites, which included restricted mesohabitats (e.g., streams, rock out crops, cliffs), all of which were examined for lichens. Each of the mesohabits contained a number of microhabitats (e.g., snags, tree bases, calcareous rocks), all of which we attempted to examine. Selva $(1999,2003)$ also used this method for sampling lichens and refers to it as an "intelligent meander", as it allows more time to be spent in areas with a higher number of lichen species.

Lichen abundance was determined by the number of sampling sites in which each species occurred. A voucher specimen of each taxon was collected at each site that was inventoried.

\section{Lichen identification}

Vouchers were identified using a stereo or compound microscope and chemical spot tests with paraphenylenediamine in ethyl alcohol, nitric acid, sodium hypochlorite, $10 \%$ potassium hydroxide, and Lugol's iodine (Brodo et al. 2001). Chemistry was further examined using a long-wave ultraviolet light chamber. Specimens that could not be reliably identified by morphology, spot tests, or ultraviolet light were analyzed for secondary chemistry using thin-layer chromatography following Culberson and Kristinsson (1970) and Orange et al. (2001). Images were captured using a Panasonic Lumix DMC-ZS20 digital camera with a $20 \times$ optical zoom.

Voucher specimens are housed at the Biodiversity Institute of Ontario Herbarium (OAC) at the University of Guelph, Guelph, Ontario, and at the William and Lynda Steere Herbarium of the New York Botanical Garden (New York) (acronyms follow the Registry of Biological Repositories, http://www.biorepositories .org).

\section{Results}

One hundred and fifty-four species of lichen-forming and allied fungi in 79 genera are reported from the Copeland Forest Resources Management Area. Sixtyfive species $(42 \%)$ are macrolichens, and 89 species (58\%) are microlichens (crustose). Of the 65 macrolichens, 29 species $(19 \%$ of the 154) are fruticose in form, and 36 species (23\% of the 154 ) are foliose. One hundred and thirty-five ( $88 \%$ of the 154$)$ lichen species have green algae as their primary photobiont, 5 species (3\%) have cyanobacteria as their primary photobiont, and 14 species $(9 \%)$ are non-lichenized fungi traditionally treated with lichens. Fifteen species $(10 \%)$ are calicioids (stubble lichens and allied fungi).

Lichen species in the Copeland Forest Resources Management Area with a provincial rank of S1 (critically imperilled) or S2 (imperilled) assigned by the Ontario Ministry of Natural Resources are (Newmaster et al., in press): Arthonia byssacea, Arthonia ruana, Chaenothecopsis pusiola, Cresponea chloroconia, Pachyphiale fagicola, and Placynthiella uliginosa. Lichens from the Copeland Forest that have a low provincial rank but the rank is uncertain (either $\mathrm{S} 1 \mathrm{~S} 3$ or S2S3) are: Anisomeridium polypori, Chaenothecopsis debilis, Porpidia cinereoatra, and Stenocybe major.

Twenty species found in the study area were designated rare in southern Ontario by Wong and Brodo (1992): Anisomeridium polypori, Arthonia byssacea, Arthonia ruana, Calicium trabinellum, Chaenothecopsis 
TABLE 1. Coordinates and habitat descriptions for the 24 lichen sampling sites in the Copeland Forest Resources Management Area north of Barrie, Ontario. See Figure 1 for locations.

\begin{tabular}{|c|c|c|c|}
\hline Site no. & Latitude $\left({ }^{\circ} \mathrm{N}\right)$ & Longitude $\left({ }^{\circ} \mathrm{W}\right)$ & Habitat \\
\hline$\overline{1}$ & $44^{\circ} 35^{\prime} 13^{\prime \prime}$ & $79^{\circ} 40^{\prime} 57^{\prime \prime}$ & $\begin{array}{l}\text { Mature second-growth forest around main parking lot. Tree cover } \\
\text { dominated by Fraxinus americana, Picea sp., and Quercus rubra. }\end{array}$ \\
\hline 2 & $44^{\circ} 35^{\prime} 00^{\prime \prime}$ & $79^{\circ} 40^{\prime} 56^{\prime \prime}$ & $\begin{array}{l}\text { Wetland along river. Moist. Protected from wind and exposed to light. } \\
\text { Tree cover dominated by Alnus incana ssp. rugosa, Picea spp., Pinus } \\
\text { strobus, Thuja occidentalis, and many snags. }\end{array}$ \\
\hline 3 & $44^{\circ} 34^{\prime} 49^{\prime \prime}$ & $79^{\circ} 41^{\prime} 15^{\prime \prime}$ & $\begin{array}{l}\text { Mature second-growth forest around pond. Tree cover dominated by } \\
\text { Acer rubrum, Acer saccharum, Fagus grandifolia, Thuja occidentalis, } \\
\text { and Tsuga canadensis. }\end{array}$ \\
\hline 4 & $44^{\circ} 33^{\prime} 57^{\prime \prime}$ & $79^{\circ} 40^{\prime} 36^{\prime \prime}$ & $\begin{array}{l}\text { Semi-exposed fields and forest. Tree cover dominated by Pinus strobus, } \\
\text { Pinus sylvestris, Populus tremuloides, Quercus rubra, and Rhus typhina. }\end{array}$ \\
\hline 5 & $44^{\circ} 34^{\prime} 03^{\prime \prime}$ & $79^{\circ} 41^{\prime} 02^{\prime \prime}$ & $\begin{array}{l}\text { Small beaver pond. Tree cover dominated by Pinus resinosa, Abies } \\
\text { balsamea, Acer rubrum, and Quercus rubra. }\end{array}$ \\
\hline 6 & $44^{\circ} 34^{\prime} 01^{\prime \prime}$ & $79^{\circ} 40^{\prime} 27^{\prime \prime}$ & $\begin{array}{l}\text { Wetland with pond. Tree cover dominated by Acer saccharum, Fraxinus } \\
\text { sp., Quercus rubra, and Thuja occidentalis. }\end{array}$ \\
\hline 7 & $44^{\circ} 33^{\prime} 56^{\prime \prime}$ & $79^{\circ} 49^{\prime} 20^{\prime \prime}$ & $\begin{array}{l}\text { Mature second-growth forest. Exposed rock. Tree cover dominated by } \\
\text { Acer saccharum and Fagus grandifolia. }\end{array}$ \\
\hline 8 & $44^{\circ} 35^{\prime} 21^{\prime \prime}$ & $79^{\circ} 40^{\prime} 04^{\prime \prime}$ & $\begin{array}{l}\text { Mature second-growth mixedwoods along the Coldwater river. Tree } \\
\text { cover dominated by Abies balsamea, Acer saccharum, Betula } \\
\text { alleghaniensis, Betula papyrifera, Thuja occidentalis, Tilia americana, } \\
\text { and Tsuga canadensis. }\end{array}$ \\
\hline 9 & $44^{\circ} 34^{\prime} 44^{\prime \prime}$ & $79^{\circ} 41^{\prime} 57^{\prime \prime}$ & $\begin{array}{l}\text { Exposed grassy area along the Coldwater river. Exposed rocks. Tree } \\
\text { cover dominated by Populus balsamifera, Picea glauca, Fraxinus } \\
\text { americana, Populus grandidentata, and Malus sp. }\end{array}$ \\
\hline 10 & $44^{\circ} 34^{\prime} 06^{\prime \prime}$ & 79॰39'38" & $\begin{array}{l}\text { Mature and dry second-growth forest with only deciduous trees. Tree } \\
\text { cover dominated by Acer saccharum, Fagus grandifolia, and Fraxinus } \\
\text { americana. }\end{array}$ \\
\hline 11 & $44^{\circ} 34^{\prime} 37^{\prime \prime}$ & 79³9'33" & $\begin{array}{l}\text { Young forest with a few old trees and a rock wall. Tree cover dominated } \\
\text { by Acer saccharum, Betula papyrifera, and Pinus sylvestris. }\end{array}$ \\
\hline 12 & $44^{\circ} 34^{\prime} 3^{\prime \prime}$ & 79॰39'3" & $\begin{array}{l}\text { Exposed rolling hills with sandy soil. Ground cover dominated by } \\
\text { Cladonia subgenus Cladina. Tree cover dominated by Acer saccharum, } \\
\text { Pinus strobus, Pinus sylvestris, Quercus rubra, and Rhus typhina. }\end{array}$ \\
\hline 13 & $44^{\circ} 33^{\prime} 2^{\prime \prime}$ & $79^{\circ} 43^{\prime} 4^{\prime \prime}$ & $\begin{array}{l}\text { Treed wetland and swamp. Numerous stumps and snags. Tree cover } \\
\text { dominated by Abies balsamea, Acer rubrum, Alnus incana ssp. rugosa, } \\
\text { Picea glauca, Pinus strobus, and Thuja occidentalis. }\end{array}$ \\
\hline 14 & $44^{\circ} 32^{\prime} 54^{\prime \prime}$ & $79^{\circ} 43^{\prime} 30^{\prime \prime}$ & Exposed wetland. Cement culvert. Decorticated snags. \\
\hline 15 & $44^{\circ} 34^{\prime} 01^{\prime \prime}$ & $79^{\circ} 42^{\prime} 41^{\prime \prime}$ & $\begin{array}{l}\text { Mature second-growth mixedwood forest. Tree cover dominated by } \\
\text { Acer rubrum, Acer saccharum, Pinus strobus, Populus spp., and Tsuga } \\
\text { canadensis. }\end{array}$ \\
\hline 16 & $44^{\circ} 34^{\prime} 31^{\prime \prime}$ & $79^{\circ} 42^{\prime} 21^{\prime \prime}$ & $\begin{array}{l}\text { Mature and moist second-growth forest around pond and wetland. Tree } \\
\text { cover dominated by Acer saccharum, Acer rubrum, Betula alleghaniensis, } \\
\text { Betula papyrifera, and Tsuga canadensis. }\end{array}$ \\
\hline 17 & $44^{\circ} 35^{\prime} 32^{\prime \prime}$ & $79^{\circ} 40^{\prime} 13^{\prime \prime}$ & $\begin{array}{l}\text { Mature second-growth forest on either side of a small stream. Tree cover } \\
\text { dominated by Acer rubrum, Acer saccharum, Fagus grandifolia, Pinus } \\
\text { strobus, and Tsuga canadensis. }\end{array}$ \\
\hline 18 & $44^{\circ} 34^{\prime} 58^{\prime \prime}$ & $79^{\circ} 39^{\prime} 43^{\prime \prime}$ & $\begin{array}{l}\text { Mature second-growth forest on steep ridges. Tree cover dominated by } \\
\text { Acer saccharum, Fagus grandifolia, Quercus rubra, and Tsuga canadensis. }\end{array}$ \\
\hline 19 & $44^{\circ} 34^{\prime} 45^{\prime \prime}$ & $79^{\circ} 40^{\prime} 06^{\prime \prime}$ & $\begin{array}{l}\text { Moist valley. Mature second-growth trees around small pond. Exposed } \\
\text { sandy soil. Tree cover dominated by Betula alleghaniensis, Betula } \\
\text { papyrifera, Fagus grandifolia, and Tsuga canadensis. }\end{array}$ \\
\hline 20 & $44^{\circ} 34^{\prime} 10^{\prime \prime}$ & $79^{\circ} 41^{\prime} 29^{\prime \prime}$ & $\begin{array}{l}\text { Wet, swampy. Mature trees. Tree cover dominated by Abies balsamea, } \\
\text { Acer rubrum, Populus balsamifera, and Thuja occidentalis. }\end{array}$ \\
\hline 21 & $44^{\circ} 33^{\prime} 32^{\prime \prime}$ & $79^{\circ} 41^{\prime} 22^{\prime \prime}$ & $\begin{array}{l}\text { Grassy field with sandy soil. Surrounding tree cover dominated by Acer } \\
\text { saccharum, Fraxinus americana, Pinus resinosa, Pinus sylvestris, and } \\
\text { Quercus rubra. }\end{array}$ \\
\hline 22 & $44^{\circ} 33^{\prime} 30^{\prime \prime}$ & $79^{\circ} 41^{\prime} 41^{\prime \prime}$ & $\begin{array}{l}\text { Mature second-growth forest on either side of an old decommissioned } \\
\text { road. Tree cover dominated by Acer saccharum, Quercus rubra, and } \\
\text { Tsuga canadensis. }\end{array}$ \\
\hline 23 & $44^{\circ} 33^{\prime} 06^{\prime \prime}$ & $79^{\circ} 41^{\prime} 58^{\prime \prime}$ & $\begin{array}{l}\text { Moist, young forest. Tree cover dominated by Abies balsamea and } \\
\text { Thuja occidentalis. }\end{array}$ \\
\hline 24 & $44^{\circ} 34^{\prime} 39^{\prime \prime}$ & $79^{\circ} 40^{\prime} 40^{\prime \prime}$ & $\begin{array}{l}\text { Mature second-growth forest. Tree cover dominated by Acer saccharum, } \\
\text { Betula papyrifera, Fraxinus spp., Tilia americana, and Tsuga canadensis. }\end{array}$ \\
\hline
\end{tabular}


debilis, Chaenothecopsis pusiola, Cladonia digitata, Cladonia parasitica, Cresponea chloroconia, Multiclavula mucida, Parmeliopsis hyperopta, Phaeocalicium polyporaeum, Phaeocalicium populneum, Placynthiella uliginosa, Sphinctrina anglica, Stenocybe pullatula, Stenocybe major, Stereocaulon tomentosum, Usnea subfloridana, and Vulpicida pinastri. One species found in the Copeland Forest, Pachyphiale fagicola (Figure 2D), was designated very rare by Wong and Brodo (1992).

We also recorded 25 species that were not listed by Wong and Brodo (1992) in southern Ontario: Acarospora moenium, Arthonia caudata, Arthonia helvola, Bellemerea cinereorufescens, Biatora chrysantha, Caloplaca pyracea, Candelariella lutella, Chaenotheca xyloxena, Chaenothecopsis exilis, Cladonia ochrochlora, Dictyocatenulata alba, Illosporiopsis christiansenii, Lecania croatica, Lecidea sarcogynoides, Lepraria elobata, Melanelixia fuliginosa, Micarea micrococca, Violella fucata, Peltigera extenuata, Phlyctis speirea, Porpidia cinereoatra, Protoparmelia hypotremella,
Ropalospora viridis, Xanthomendoza ulophyllodes, and Xanthoparmelia angustiphylla.

Based on a current, but unpublished, lichen list for Ontario, two of the species found in the Copeland Forest are new records for the province: Lecidea sarcogynoides and Micarea micrococca (Figure 2A-B). Lecidea sarcogynoides is also a new Canadian record, based on an unpublished national microlichen checklist that is currently being developed (Brodo, personal communication, May 2012). Micarea micrococca is reported for the first time in Canada. It was previously collected in Newfoundland, Newfoundland and Labrador. Three species found during our study are reported for the first time in Ontario: Bellemerea cinereorufescens (Figure 2C), Phlyctis speirea, and Xanthoparmelia angustiphylla. Bellemerea cinereorufescens is known from two previous collections in the province and Phlyctis speirea and Xanthoparmelia angustiphylla are both known from one previous collection. Candelariella lutella is collected and reported for the second time in Ontario and the third time in Canada.

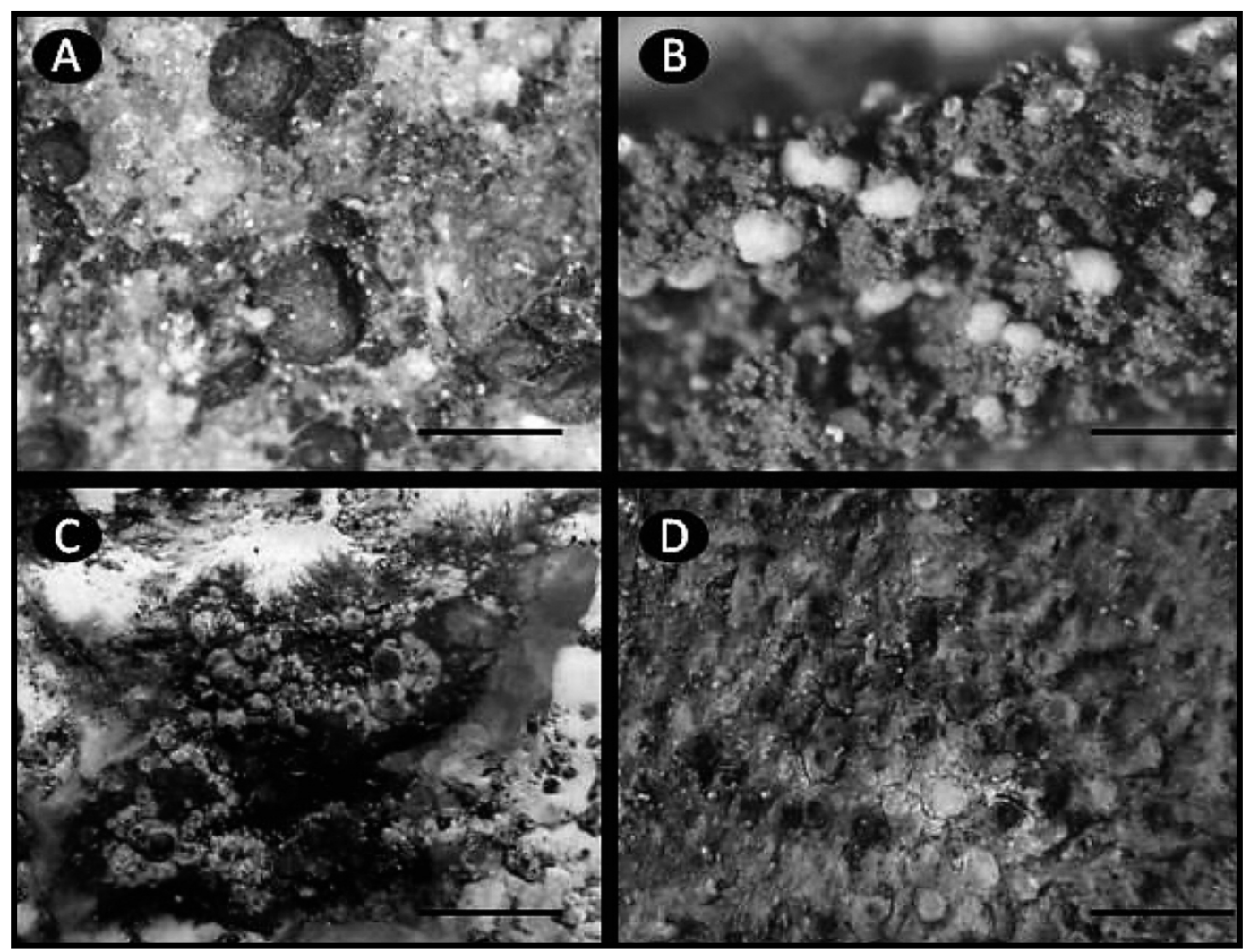

Figure 2. A and B are lichen species new to Ontario. A. Lecidea sarcogynoides, scale $=0.6 \mathrm{~mm}$, McMullin 9106, New York. B. Micarea micrococca, scale $=0.4 \mathrm{~mm}$, McMullin 9150, NY. C. Bellemerea cinereorufescens, scale $=2.5 \mathrm{~mm}$, McMullin 8959, OAC. D. Pachyphiale fagicola, scale $=1.5 \mathrm{~mm}$, McMullin 9136, OAC. 
Of the 154 taxa found during this study, none occurred in all of the 24 study sites examined in the Copeland Forest. Nineteen species were located in 7 or more sites, while 68 species were found at only a single site.

Substrate-specific species were found colonizing the following substrata exclusively: deciduous trees (26 species), rocks (21 species), snags (19 species), soil (15 species), coniferous trees (7 species), stumps (6 species), Acer saccharum ( 5 species), cement (5 species), Populus balsamifera (3 species), Quercus rubra (3 species), Rhus typhina (3 species), Thuja occidentalis (3 species), Abies balsamea (2 species), Betula alleghaniensis (2 species), Pinus strobus (2 species), Acer rubrum (1), Alnus incana ssp. rugosa (1 species), and a polypore (1 species).

Within the 24 sites in the Copeland Forest (Table 1), the highest number of lichen species was in site numbers 2 (42 species), 12 (40 species), 16 (40 species), 8 (37 species), 9 (38 species), and 13 (33 species).

\section{Annotated species list}

The annotated checklist presented below is arranged alphabetically by genus and species. Authority abbreviations follow Brummitt and Powell (1996). Taxonomy follows Esslinger (2012). In cases where the list deviates from Esslinger (2012), it reflects the taxonomic opinions of the authors.

Non-lichenized fungi treated with lichens are preceded by a dagger $(\dagger)$. Species preceded by an asterisk $(*)$ are reported (published) for the first time in Ontario. Species preceded by two asterisks $(* *)$ were collected for the first time in Ontario.

$\mathrm{S}$ and $\mathrm{G}$ ranks are conservation status ranks assigned by the Ontario Ministry of Natural Resources that are not legal determinations. S ranks are provincial designations and $\mathrm{G}$ ranks are global designations. Conservation status is designated by a number between 1 and 5: $1=$ critically imperilled, $2=$ imperilled, $3=$ vulnerable, 4 = apparently secure, 5 = secure, $\mathrm{U}=$ "unrankable" due to a lack of information, ? = rank uncertain, $\mathrm{NR}=$ reported but without verification or persuasive documentation (Ontario Ministry of Natural Resources 2012).

Frequency designations for species in southern Ontario follow the $\mathrm{S}$ and $\mathrm{G}$ ranks and are non-legal determinations by Wong and Brodo (1992). Their designations are based on the number of counties (36 total) a species was collected in: very rare $=1$ county, rare $=2$ or 3 counties, frequent $=4$ or 5 counties, common $=6$ to 8 counties, very common $=9$ to 36 counties.

The site number identifying the site in which each species was collected is in bold. The subtrate that each specimen was growing on follows the site number.

Collection numbers of RTM follow the substrates of each specimen and are housed at OAC unless stated otherwise.
Acarospora fuscata (Schrad.) Arnold: 12 - granitic rock, 8690, 8960.16 - granitic rock, 8537. S5, G5; very common (Wong and Brodo 1992).

Acarospora moenium (Vain.) Räsänen: 9 - cement culvert over water, 9139 (NY, OAC). SNR, GNR; not reported by Wong and Brodo (1992).

Amandinea dakotensis (H. Magn.) P. May \& Sheard: 12 - Rhus typhina, 8878. S4, GNR; infrequent (Wong and Brodo 1992).

Amandinea punctata (Hoffm.) Coppins \& Scheid.: 4 - Quercus rubra, 9155 (NY). 6 - snag, 9252. S5, G5; very common (Wong and Brodo 1992).

Anisomeridium polypori (Ellis \& Everh.) M.E. Barr: 10 - deciduous tree, 9148 (NY, OAC). 17 Betula alleghaniensis, 9144 (NY). 20 - Thuja occidentalis, 9146 (NY). S2S3, GNR; rare (Wong and Brodo 1992).

Arthonia byssacea (Weigel) Almq.: 8 - snag, 9113 (NY, OAC). S1S2, GNR; rare (Wong and Brodo 1992).

Arthonia caesia (Flot.) Körb.: 1 - Fraxinus, 8798. 4 Quercus rubra, 8502.9-Prunus, 8501; Malus, 8770. S5, G4G5; common (Wong and Brodo 1992).

Arthonia caudata Willey: 4 - Pinus strobus, 8508. 12 - Pinus strobus, 9280. SNR, GNR; not reported by Wong and Brodo (1992).

Arthonia helvola (Nyl.) Nyl.: 2 - Betula alleghaniensis, 8509. 16 - Betula alleghaniensis, 8503. SNR, GNR; not reported by Wong and Brodo (1992).

Arthonia ruana. Massal.: 16 - Acer saccharum, 9123. S1, GNR; rare (Wong and Brodo 1992).

Aspicilia cinerea (L.) Körb.: 12 - siliceous rock, 8731. S4S5, G5; frequent(Wong and Brodo 1992).

Bacidia schweinitzii (Fr. ex Tuck.). Scheid.: 8 -Acer saccharum, 8504. 19 - Fagus grandifolia snag, 8732. 20 - Thuja occidentalis, 9224. 23 - Thuja occidentalis, 9151 (NY, OAC). S5, G4G5; common (Wong and Brodo 1992).

*Bellemerea cinereorufescens (Ach.) Clauzade \& Cl. Roux: 12 - siliceous rock, 8959. SU, GNR; not reported by Wong and Brodo (1992).

Biatora chrysantha (Zahlbr.) Printzen: 13 - soil, 9132 (NY). SNR, GNR; not reported by Wong and Brodo (1992).

Bilimbia sabuletorum (Schreber) Arnold: 20 - Thuja occidentalis, 8979. S5, G4G5; common (Wong and Brodo 1992).

Buellia erubescens Arnold: 2 - Alnus, 8505.9-Malus, 8734. 20 - Acer rubrum, 8733. SNR, G3G5; frequent (Wong and Brodo 1992).

Calicium trabinellum (Ach.) Ach.: 13 - lignicolous, charred stump, 8627; lignicolous, stump, 8080. S4S5, G4G5; rare (Wong and Brodo 1992).

Caloplaca cerina (Ehrh. ex Hedw.) Th. Fr.: 8 - snag, 8843. 9 - Populus balsamifera, 8723, 8783. S5, G5; frequent (Wong and Brodo 1992). 
Caloplaca feracissima H. Magn.: 14 - cement culvert over water, 9149 (NY, OAC). 16 - cement culvert over water, 9154 (NY). S5, G5?; common (Wong and Brodo 1992).

Caloplaca holocarpa (Hoffm. ex Ach.) A.E. Wade: 9 cement culvert over water, 8515. S5, G5; common (Wong and Brodo 1992).

Caloplaca pyracea (Ach.) Th. Fr.: 9 - Populus balsamifera, 8510, 8722. S5, G5; included with C. holocarpa by Wong and Brodo (1992).

Candelaria concolor (Dicks.) Stein: 1 - Fraxinus, 8794. 3 - deciduous snag, 8826. 4 - Quercus rubra, 8853.8 - Acer saccharum, 8507; snag, 8842; Abies balsamea, 8848. 9-Malus, 8767, 8777. 11 - Acer, 8860.17 - Acer saccharum, 8840. S5, G5; very common (Wong and Brodo 1992).

Candelariella aurella (Hoffm.) Zahlbr.: 9 - cement culvert over water, 8506.16 - cement culvert over water, 8998. S5, G5?; common (Wong and Brodo 1992).

Candelariella efflorescens R.C. Harris \& W.R. Buck: 1 - Quercus rubra, 9138 (NY). 9 - Populus balsamifera, 8764. 13 - Prunus, 8511, 8512; Acer rubrum, 8994; Thuja occidentalis snag, 8995. 16 - Acer rubrum, 8994. 23 - Thuja occidentalis, 8997. S5, G4G5; common (Wong and Brodo 1992).

Candelariella lutella (Vain.) Räsänen: 9 - Populus balsamifera, 9281. SNR, GNR; not reported by Wong and Brodo (1992).

Candelariella vitellina (Hoffm.) Müll. Arg.: 12 - granitic rock, 8762. S5, G5; very common (Wong and Brodo 1992).

Cetrelia olivetorum (Nyl.) W.L.Culb. \& C.F.Culb.: 1 Quercus rubra, 9258. 2 - deciduous snags, 8513, 8514. S4, G3G5; frequent (Wong and Brodo 1992).

Chaenotheca ferruginea (Turner ex Sm.) Mig.: 2 lignicolous snag, 8078. 8 - Thuja occidentalis, 8735. 17 - lignicolous snag, 8070. S4, G4G5; infrequent (Wong and Brodo 1992).

Chaenotheca furfuracea (L.) Tibell: 3 - fine tree roots in moist cavern, 8086.19 - tree roots in a moist, sheltered environment, 9020. S4, G4G5; infrequent (Wong and Brodo 1992).

Chaenotheca xyloxena Nádv.: 17 - decorticated snags, 8060, 8073, 8075. SNR, GNR; not reported by Wong and Brodo (1992).

$\dagger$ Chaenothecopsis debilis (Turner \& Borrer ex Sm.) Tibell: 2 - Thuja occidentalis snag, 8094. 8 wooden fence post, 8087.10 - lignicolous snag, 8091. S2S3, GNR; rare (Wong and Brodo 1992).

$\uparrow$ Chaenothecopsis exilis Tibell: 16 - decorticated snag, 8068. 17 - decorticated snag, 8071. SNR, GNR; not reported by Wong and Brodo (1992). $\dagger$ Chaenothecopsis pusiola (Ach.) Vain.: 17 - decorticated snags, 8059, 8074. S1S2, GNR; rare Wong and Brodo (1992).

$\dagger$ Chaenothecopsis spp.: 2 - decorticated snags, 9287 9288. 8 - decorticated snag, 8058. 9- decorticated snag, 9246.13 - decorticated snag, 8093. 17 - decorticated snag, 8076 . Notes: These specimens appear to be undescribed species.

Cladonia acuminata (Ach.) Norrl.: 4 - sandy soil, 8700. SNR, G5?; infrequent (Wong and Brodo 1992).

Cladonia cenotea (Ach.) Schaer.: 13 - stump, 8518. S5, G5; frequent (Wong and Brodo 1992).

Cladonia chlorophaea (Flörke ex Sommerf.) Spreng.: 2 - Thuja occidentalis snag, 8541; stump, 9271. 4 - sandy soil, 9264.7 - base of Fagus grandifolia, 9277. 8 - Thuja occidentalis, 9265.10 - the base of Acer saccharum, 8519. 11 - soil, 9275. 12 - sandy soil, 9272.13 - stump, 9273. 15 - soil, 9274. 16 - Acer saccharum, 9270; fence post, 9276.19 - sandy soil, 9266, 9268, 9269. S5, GU; very common (Wong and Brodo 1992).

Cladonia coniocraea (Flörke) Spreng.: 2 - coarse woody debris, 8520; Betula papyrifera snag, 8523; Tsuga canadensis snag, 8788. 8 - Tsuga canadensis snag, 8524; stump; 8525.10 - coarse woody debris, 8521.13 - stump, 8522. S5, G5; very common (Wong and Brodo 1992).

Cladonia cristatella Tuck.: 2 - snag, 8888. 6 - snag, 8526. 12 - soil, 8527-8529. 13 - decorticated stump, 8940. 15 -soil, 8530. 16 - soil, 8701. 19 - soil, 8531. 21 - soil, 8992. S5, G5?; very common (Wong and Brodo 1992).

Cladonia digitata (L.) Hoffm.: 10 - base of an Acer saccharum, 8532. S4S5, G3G5; rare (Wong and Brodo 1992).

Cladonia fimbriata (L.) Fr.: 2 - Thuja occidentalis snag, 8789. 4 - sandy soil, 8534, 8702. 8 - Tsuga canadensis snag, 8535.10 - Acer saccharum, 8533. 13 - Acer rubrum, 8536; Prunus, 8538. 16 - Betula alleghaniensis, 8703. 19 - sandy soil, 8539, 8704. S5, G3G5; common (Wong and Brodo 1992).

Cladonia grayi G. Merr. ex Sandst.: 2 - Thuja occidentalis snag, 8540. 13 - soil, 8626. S4S5, GU; frequent (Wong and Brodo 1992).

Cladonia macilenta var. bacillaris (Ach. Schaer.: 6 decorticated coarse woody debris, 8628.10 base of an Acer saccharum, 8544. S5, G5T5; common (Wong and Brodo 1992).

Cladonia mitis Sandst.: 12 - sandy soil, 8516, 8517, 8543. 16 - soil, 8699. SNR, GNR; common Wong and Brodo (1992).

Cladonia ochrochlora Flörke: 2 - stump, 8545. 11 coarse woody debris, 9267.17 - base of a snag, 8705. 19 - coarse woody debris, $8546,8547.23$ - soil, 8990. SNR, G3G5; not reported by Wong and Brodo (1992). 
Cladonia parasitica (Hoffm.) Hoffm.: 10 - snag, 8548. SNR, G3G5; rare (Wong and Brodo 1992).

Cladonia phyllophora Hoffm.: 12 - soil, 8549, 8550, 8707; sandy soil, 8553.19 - on soil, 8706 . S5, G5; common (Wong and Brodo 1992).

Cladonia pleurota (Flörke) Schaer.: 12 - sandy soil, 8551, 8552.16 - soil, 8709. S5, G3G5; frequent (Wong and Brodo 1992).

Cladonia pyxidata (L.) Hoffm.: 4 - sandy soil, 8858. $\mathbf{8}$ - Thuja occidentalis, 8554.12 - sandy soil, 8555-8557. S5, G5; very common (Wong and Brodo 1992).

Cladonia rangiferina (L.) F.H. Wigg.: 4 - sandy soil, $8720 . \mathbf{6}$ - snag, 8558.11 - soil, 8559.12 - sandy soil, 8560-8562.16 - soil, 8712.19 - sandy soil, 8711. S5, G5; common (Wong and Brodo 1992).

Cladonia rei Schaer.: 2 - Thuja occidentalis snag, 9257. $\mathbf{4}$ - sandy soil, $8563,8714,9278.19$ - sandy soil, 8564, 8713, 9279. 21 - soil, 8982. S5, G3G5; very common (Wong and Brodo 1992).

Cladonia turgida Hoffm.: 12 - soil, 8565. 19 - soil, 8566, 8715. S5, G3G5; common (Wong and Brodo 1992).

Cladonia uncialis (L.) F.H. Wigg.: 12 - sandy soil, 8567-8569, 8708. 19 - sandy soil, 8716. S5, G4G5; frequent (Wong and Brodo 1992).

Cladonia verticillata (Hoffm.) Schaer:: 4 - sandy soil, 8519. 12 - sandy soil, $8570,8571,8857.15$ soil, 8572.16 - soil, 8717.19 - sandy soil, 8573 , 8718. S4S5, G5TNR; very common (Wong and Brodo 1992).

Coenogonium pineti (Ach.) Lücking \& Lumbsch: 5 Betula alleghaniensis, 8847. 13 - base of an Acer rubrum, 8632. 20 - Thuja occidentalis, 9225. S3, GNR; infrequent (Wong and Brodo 1992).

Cresponea chloroconia (Tuck.) Egea \& Torrente: 2 Thuja occidentalis snags, 8629, 8630. S1S2, GNR; rare (Wong and Brodo 1992).

Dictyocatenulata alba Finley \& E.F. Morris: 2 - Betula alleghaniensis, 8574. 5 - Betula alleghaniensis, 8736. SNR, GNR; not reported by Wong and Brodo (1992).

Evernia mesomorpha Nyl.: 2 - snag, 8575; Thuja occidentalis snag, 8810; Pinus strobus, 8576. 5 Abies balsamea, 8812. 8 - Abies balsamea, 8790. 9 - Prunus, 8577; Malus 8867. 10 - snag, 8951. 11 - Pinus resinosa, 8949. 12 - snag, 8802; Rhus typhina, 8578.16 - Pinus sylvestris snag, 8939. S5, G5; common (Wong and Brodo 1992).

Flavoparmelia caperata (L.) Hale: 1 - Fraxinus, 8797. 2 - Thuja occidentalis snag, 8579; snag, 8830. 8 - Thuja occidentalis, 8866. 9 - Prunus, 8580; snag, 9220.10 - snag, 8953. 13 - Prunus, 8581; Picea glauca, 8582. 16 - snag, 8862; Pinus sylvestris snag, 8938. 20 - Thuja occidentalis, 8863. S5, G5?; very common (Wong and Brodo 1992).
Flavopunctelia flaventior (Stirt.) Hale: 9 - snag, 9218. S5, G5; common (Wong and Brodo 1992).

Graphis scripta (L.) Ach.: 2 - Betula alleghaniensis, 8583. 3 - Acer saccharum, 8822. 5 - Betula alleghaniensis, 8846.8 - Acer saccharum, 8584; deciduous snag, 8883. 11 - Acer, 8585; Acer saccharum, 8874.13-Quercus rubra, 8586. 16 - Betula alleghaniensis, 8587; Acer saccharum, 8814, 8877. 18 - Acer saccharum, 8588. 22 Acer saccharum, 8980. 24 - Acer saccharum, 8986. S5, G5; very common (Wong and Brodo 1992).

Hypocenomyce friesii (Ach.) P. James \& Gotth. Scheid.: 13 - charred decorticated stump, 8589. SNR, G3G5; infrequent (Wong and Brodo 1992).

Hypocenomyce scalaris (Ach. ex Lilj.) M. Choisy: 13 - charred decorticated stumps, 8590, 8591. S5, G5; very common (Wong and Brodo 1992).

Hypogymnia physodes (L.) Nyl.: 2 - snag, 8592; Pinus strobus, 8765; Thuja occidentalis snag, 8809; deciduous snag, 8837. 5 - Abies balsamea, 8813 . $\mathbf{8}$ - Abies balsamea, 8593. 11 - Pinus resinosa, 8948. 13 - decorticated stump, 8943.16 - Pinus sylvestris snag, 8935. 17 - snag, 8801. S5, G5; very common (Wong and Brodo 1992).

$\dagger$ Illosporiopsis christiansenii (B. L. Brady \& D. Hawksw.) D. Hawks:1 - Physcia stellaris, 8631; 9 - Physcia stellaris, 8774, 8954. SNR, GNR; not reported by Wong and Brodo (1992).

Imshaugia aleurites (Ach.) S.F. Mey.: $\mathbf{2}$ - branch of Thuja occidentalis snag, 8594. S4S5, G5, frequent (Wong and Brodo 1992).

$\dagger$ Julella fallaciosa (Arnold) R.C. Harris: 2 - Betula papyrifera, 9263.8 - Acer saccharum, 8962. 10 - Acer saccharum, 8738. 11 - Acer saccharum, 8963. 16 - Acer saccharum, 8964; Betula papyrifera 9262. 17 - Acer saccharum, 8737. 18 Acer saccharum, 8966. 22 - Acer saccharum, 8967. 24 - Acer saccharum, 8965. SNR, GNR; frequent (Wong and Brodo 1992).

Lecania croatica (Zahlbr.) Kotlov: 2 - Acer saccharum, 8970. 8 - Acer saccharum, 8971, 8972.9 - snag, 8968. 19 - snag, 8969. SNR, GNR; not reported by Wong and Brodo (1992).

Lecania naegelii (Hepp) Diederich \& v.d. Boom: 1 Fraxinus, 8595, 8596. 4 - Populus balsamifera, 8597. 9 - Populus balsamifera, 8598, 8739; Fraxinus, 9254. S2S4, GNR; infrequent (Wong and Brodo 1992).

Lecanora albella (Pers.) Ach. var. rubescens (Imshaug \& Brodo) Lumbsch: 13 - Pinus strobus, 8961. SNR, G4G5TNR; frequent (Wong and Brodo 1992).

Lecanora caesiorubella Ach. subsp. caesiorubella: 20 - Acer rubrum, 8740. S4S5, G4G5TNR; frequent (Wong and Brodo 1992).

Lecanora hybocarpa (Tuck.) Brodo: 2 - snag, 9250.9 - Populus balsamifera, 8599; Fraxinus, 9255. 
13 - deciduous bark, 8600. S4S5, G5; common (Wong and Brodo 1992).

Lecanora meridionalis H. Magn.: 13 - stump, 9001. SNR, GNR; infrequent (Wong and Brodo 1992).

Lecanora muralis (Schreb.) Rabenh.: 12 - rock, 9104 (NY). S5, G5; common (Wong and Brodo 1992).

Lecanora polytropa (Hoffm.) Rabenh.: 9 - siliceous rock, 8820. S5, G5; frequent (Wong and Brodo 1992).

Lecanora pulicaris (Pers.) Ach.: 2 - Pinus strobus, 8729. 4 - Pinus strobus, 8845. 5 - Abies balsamea, 8811; snag, 8851.12 - Pinus strobus, 8601. 13 - Picea glauca, 8602, 8724. S5, G5; common (Wong and Brodo 1992).

Lecanora rugosella Zahlbr.: 2 - Thuja occidentalis snags, 8603, 8871. 8 - Thuja occidentalis, 8604. 20 - Thuja occidentalis, 9223. S4S5, G5?; frequent (Wong and Brodo 1992).

Lecanora symmicta (Ach.) Ach.: 1 - Fraxinus, 8605. 2 - Pinus strobus, 8730. 5 - snag, 8852.9 - Prunus, 8606; Populus balsamifera, 8784. 13 Picea glauca, 8725; stump, 8941, 8975. 16 Pinus sylvestris snag, 8936. 17 - snag, 8800. S5, G5; common (Wong and Brodo 1992).

Lecanora thysanophora R.C. Harris: 1 - Fraxinus, 8796. 2 - Thuja occidentalis snag, 8607; Acer, 8608. 8 - Acer saccharum, 8609. 11 - Acer saccharum, 8610. 16 - Acer rubrum, 8611; Acer saccharum, 8741. 17 -Acer rubrum, 8612. 24 - Acer saccharum, 8977. S5, GNR; frequent (Wong and Brodo 1992).

**Lecidea sarcogynoides Körb.: 12 - rock, 9106 (NY). SNR GNR; not reported by Wong and Brodo (1992).

Lepraria caesiella R.C. Harris: 8 - Tsuga canadensis, 9116 (OAC, NY). 20 - Abies balsamea, 9145 (NY). SNR, GNR; frequent (Wong and Brodo 1992).

Lepraria elobata Tønsb.: 3 - Fagus grandifolia, 9129 (OAC, NY). 8 - Tsuga canadensis, 9118 (NY). SU, GNR; not reported by Wong and Brodo (1992).

Lepraria finkii (B. de Lesd.) R.C. Harris: 1 - Picea, 9134 (NY). 2 - Betula alleghaniensis, 9131.8 - Thuja occidentalis, 9121.13 - Quercus rubra, 9251.16 - Thuja occidentalis, 9124.18-Acer saccharum, 9256. 19 - snag, 9112. S5, GNR; common (Wong and Brodo 1992).

$\dagger$ Leptorhaphis epidermidis (Ach.) Th. Fr.: 4 - Betula papyrifera, 8613. S4, GNR; infrequent (Wong and Brodo 1992).

Megalaria laureri (Hepp ex Th. Fr.) Hafellner: 19 Fagus grandifolia snag, 9110 (NY, OAC). SNR, GNR; infrequent (Wong and Brodo 1992).

Melanelia sorediata (Ach.) Goward \& Ahti: 12 - rock, 8617,8618 . S5, GNR; infrequent (Wong and Brodo 1992).
Melanelixia fuliginosa (Fr. ex Duby) O. Blanco et al.: 2 - snag, 8614; Pinus strobus, 8728.16-Acer rubrum, 8615, 8616. S3, G5; not reported by Wong and Brodo (1992).

Melanelixia subaurifera (Nyl.) O. Blanco et al: 1 Fraxinus americana, 8884.3 - deciduous snag, 8823. 6 - snag, 8792. 9 - Malus, 8772, 8779, 8869; on Populus balsamifera, 8787; Prunus, 8805; Picea glauca, 8957. 11 - Acer, 8781; Acer saccharum, 8818. 12 - Pinus strobus, 8624; Rhus typhina, 8880. 13 - stump, 8942. 16 Pinus sylvestris snag, 8937; Acer saccharum, 8876. 24 - Acer saccharum, 8985. S5, G3G5; very common (Wong and Brodo 1992).

**Micarea micrococca (Körb.) Gams ex Coppins: 8 - snag, 9150 (NY). SNR, GNR; not reported by Wong and Brodo (1992).

Micarea peliocarpa (Anzi) Coppins \& R. Sant.: 2 snag, 8619; Thuja occidentalis snag, 8620. 16 - Thuja occidentalis, 8621. S4S5, G4G5; frequent (Wong and Brodo 1992).

Micarea sp.: 16 - wooden fence post, 9157 (NY).

Multiclavula mucida (Fr.) R. Petersen: 15 - moist fallen $\log , 8622$. SU, GNR; rare (Wong and Brodo 1992).

$\uparrow$ Mycocalicium subtile (Pers.) Szatala: $\mathbf{2}$ - decorticated snag, 8092, 8096, 9286. 5 - decorticated snag, $8084,8095.13$ - decorticated snag, 8083, 8088. 16 - decorticated snag, 8069. S4S5, G4G5; infrequent (Wong and Brodo 1992).

Myelochroa aurulenta (Tuck.) Elix \& Hale: 8 -Acer saccharum, 8623, 8625. 17 - Acer saccharum, 8742. 22 - Acer saccharum, 8976. S5, G5?; common (Wong and Brodo 1992).

Ochrolechia arborea (Kreyer) Almb.: 1 - Quercus rubra, 9137 (NY). 2 - snag, 8633. 5 - snag, 8850. 6 - snag, 8634. S4S5, GNR; common (Wong and Brodo 1992).

Pachyphiale fagicola (Hepp) Zwackh: 1 - Quercus rubra, 9136. S1, GNR; very rare (Wong and Brodo 1992).

Parmelia sulcata Taylor: 1 - Fraxinus americana, 8886. 2 - snag, 8829; deciduous snag, 8838; Thuja occidentalis snag, 8873.3 - deciduous snag, 8825. 6 - snag, 8793. 9 - Malus, 8768, 8776, 8868; Prunus, 8804; Fraxinus, 8832; Picea glauca, 8956. 10 - snag, 8952. 11 - Acer saccharum, 8817; Pinus resinosa, 8947. 12 - Pinus strobus, 8635; snag, 8803; Rhus typhina, 8881. 13 - Acer rubrum, 8636; stump, 8944. 16 snag, 8637; deciduous snag, 8638; Pinus sylvestris snag, 8934. S5, G5; very common (Wong and Brodo 1992).

Parmeliopsis ambigua (Wulfen) Nyl.: 12 - snag, 8639; Rhus typhina snag, 8640. S5, G3G5; infrequent (Wong and Brodo 1992). 
Parmeliopsis hyperopta (Ach.) Arnold: 12 - snag, 8743; Rhus typhina snag, 8641. S5, G3G5; rare (Wong and Brodo 1992).

Peltigera didactyla (With.) J.R. Laundon: 4 - sandy soil, 8744. 19 - sandy soil, 8642. S5, G5; common (Wong and Brodo 1992).

Peltigera extenuata (Vain.) Lojka: 4 - sandy soil, 8643, 8644, 8743. Notes: Included with Peltigera didactyla in the past. SNR, GNR; see Peltigera didactyla in Wong and Brodo (1992).

Peltigera lepidophora (Nyl. ex Vain.) Bitter: 4 - sandy soil, 8746. 19 - sandy soil, 8771. 21 - on soil, 8991. S4, G4; frequent (Wong and Brodo 1992).

Peltigera praetextata (Flörke ex Sommerf.) Zopf: 8 base of an Acer saccharum, 8645. 19 - decomposing log, 8646; soil, 8747. 24 - base of an Acer saccharum, 8987. S5, G3G5; common (Wong and Brodo 1992).

Peltigera rufescens (Weiss) Humb.: 4 - sandy soil, 8647, 8648. S5, G5; common (Wong and Brodo 1992).

Pertusaria macounii (I.M. Lamb) Dibben: 8 - Acer saccharum, 8748, 8799, 9282. 24 - Acer saccharum, 8984. S4, G4G5; frequent (Wong and Brodo 1992).

$\dagger$ Phaeocalicium curtisii (Tuck.) Tibell: 1 - Rhus typhina, 8082. 2 - Rhus typhina, 8101.9-Rhus typhina, 8061.12 - Rhus typhina, 8067.16-Rhus typhina, 9247. S5, GNR; common (Wong and Brodo 1992).

$\dagger$ Phaeocalicium polyporaeum (Nyl.) Tibell: 3 - polypore, $8090,8102.8$ - polypore, 8098.10 - polypore, 8085.11 - polypore, 8064.17 - polypore, 9285. 19 - polypore, 8066. S3, GNR; rare (Wong and Brodo 1992).

$\uparrow$ Phaeocalicium populneum (Brond. ex Duby) A.F.W. Schmidt: 9 - Populus balsamifera, 8072. SU, GNR; rare (Wong and Brodo 1992).

Phaeophyscia adiastola (Essl.) Essl.: 4 - Quercus rubra, 8855. 11 - mossy rock, 8749. S4, G4?; very common (Wong and Brodo 1992).

Phaeophyscia pusilloides (Zahlbr.) Essl.: 1 - Fraxinus, 8763. 3 - deciduous snag, 8827. 4 - Quercus rubra, 8651. 8 - Acer saccharum, 8652; snag, 8841; Abies balsamea, 8849.9283.9-Populus balsamifera, 8750; Fraxinus, 8833.11-Acer, 8859.16-Acer rubrum, 8653; Acer saccharum, 8836. 19-Acer, 8654. S5, G5; common (Wong and Brodo 1992).

Phaeophyscia rubropulchra (Degel.) Essl.: 1 - Fraxinus, 8795; Quercus rubra, 9260. 2 -Acer, 8656. 3 - Acer saccharum, 8821.4 - Quercus rubra, 8655; Acer saccharum, 8657. 8-Acer saccharum, 8658, 8807. 9 - Malus, 8870. 13 -Acer rubrum, 8659.16-Acer saccharum, 8835. 17 - Acer saccharum, 8839. 24 - Acer saccharum, 8978. S5, G5; very common (Wong and Brodo 1992).
Phlyctis argena (Spreng.) Flot.: 2 - Thuja occidentalis snag, 8674, 8675.20 - Thuja occidentalis, 9226. S4S5, G4G5; infrequent (Wong and Brodo 1992).

*Phlyctis speirea G. Merr.: 20 - Thuja occidentalis, 8973. SNR, GNR; not reported by Wong and Brodo (1992).

Physcia adscendens (Fr.) H. Olivier: 1 - Fraxinus americana, 8885.3 - deciduous snag, 8828.4 - Quercus rubra, 8854. 8 - snag, 8844. 9 deciduous snag, 8721; Malus, 8769, 8778; Populus balsamifera, 8786; Fraxinus, 8834; Picea glauca, 8955. 12 - Rhus typhina, 8660. 13 - stump, 8945. 16 - deciduous snag, 8661; Picea glauca, 8662. S5, G5; very common (Wong and Brodo 1992).

Physcia aipolia (Ehrh. ex Humb.) Fürnr.: 1 - Fraxinus, 8663. 4-Quercus rubra, 8751.16-deciduous snag, 8752. S5, G5TNR; very common (Wong and Brodo 1992).

Physcia dubia (Hoffm.) Lettau: 16 - rock, 8664. S5, G5; common (Wong and Brodo 1992).

Physcia millegrana Degel.: 1 - Quercus rubra, 9259; Fraxinus, 8665. 3 - deciduous snag, 8824. 4 Quercus rubra, 8856. 8 - a deciduous snag, 8882; Acer saccharum, 8666. 9- Prunus, 8806; Malus, 8766, 8775; deciduous snag, 8758. 10 - snag, 8950.11 - Acer saccharum, 8875; Acer, 8667. 13 - Acer rubrum, 8668; Picea glauca, 8727. 16-Acer saccharum, 8815. 22 - on Acer saccharum, 8983. 24 - Acer saccharum, 8988. S5, G5; very common (Wong and Brodo 1992). Physcia stellaris (L.) Nyl.: 9 - Populus balsamifera, 8669; Populus grandidentata, 8753; Malus, 8780. 11 - Acer saccharum, 8816. S5, G5; very common (Wong and Brodo 1992).

Physconia detersa (Nyl.) Poelt: 9 - deciduous snag, 8757; Fraxinus, 8831. 16 - Acer saccharum, 8670, 8754. S5, G5?; very common (Wong and Brodo 1992).

Physconia enteroxantha (Nyl.) Poelt: 1 - Fraxinus, 8673. 8 - Acer saccharum, 8671, 8808. 13 Acer rubrum, 8672. S3, G3G5; infrequent (Wong and Brodo 1992).

Placynthiella uliginosa (Schrad.) Coppins \& P. James: 12 - sandy soil, 8676. S2, G5; rare (Wong and Brodo 1992).

Porpidia cinereoatra (Ach.) Hertel \& Knoph: 18 rock, 8755. S1S3, G5?; not reported by Wong and Brodo (1992).

Porpidia crustulata (Ach.) Hertel \& Knoph: 10 - rock, 9128 (NY). S5, G4G5; common (Wong and Brodo 1992).

Porpidia macrocarpa (DC.) Hertel \& A.J. Schwab: 7 rock, $9156(\mathrm{NY}) .12$ - rock, 9109 (NY, OAC). S4, G4; frequent (Wong and Brodo 1992).

Protoblastenia rupestris (Scop.) J. Steiner: 2 cement culvert over water, 9130 (NY, OAC). S5, G4G5; common (Wong and Brodo 1992). 
Protoparmelia hypotremella Herk, Spier \& V. Wirth: 20 - decorticated Thuja occidentalis branch, 9228. SNR, GNR; not reported by Wong and Brodo (1992).

Punctelia rudecta (Ach.) Krog: 1 - Quercus rubra, 9261. 2 - Thuja occidentalis snags, 8677, 8678, 8872. 8 - Acer saccharum, 8679, 8680; Thuja occidentalis, 8865.9 - deciduous snag, 8759; snag, 9219. 10 - Thuja occidentalis, 8681. 13 - Picea glauca, 8726.16-Acer, 8782; snag, 8861.20 - Thuja occidentalis, 8864.22 - Acer saccharum, 8989. 24 - Acer saccharum, 8981. S5, G5; very common (Wong and Brodo 1992). Pyxine sorediata (Ach.) Mont.: 8 - Acer saccharum, 8682. S5, G5, common (Wong and Brodo 1992).

Ramalina intermedia (Delise ex Nyl.) Nyl.: 2 - Thuja occidentalis snag, 8683. 9 - snag, 9222. S5, G4G5; common (Wong and Brodo 1992).Rinodina tephraspis (Tuck.) Herre: $\mathbf{1 0}$ - granitic rock, 9127 (NY). S4, GNR; frequent (Wong and Brodo 1992).

Ropalospora viridis (Tønsb.) Tønsb.: 16 - Populus tremuloides, 9125 (NY, OAC). 17 - snag, 9143 (NY, OAC). SNR, GNR; not reported by Wong and Brodo (1992).

Sarcogyne hypophaea (Nyl.) Arnold: 9 - siliceous rock, 8819. SU, G2G4; infrequent (Wong and Brodo 1992).

Sarcogyne regularis Körb.: 12 - calcareous rock, 8684 . S5, G5; common (Wong and Brodo 1992).

Scoliciosporum chlorococcum (Stenh.) Vězda: 12 Rhus typhina, 8879. S5, G4G5; very common (Wong and Brodo 1992).

$\dagger$ Sphinctrina anglica Nyl.: 20 - decorticated Thuja occidentalis branch, 9227. S3, GNR; rare (Wong and Brodo 1992).

$†$ Stenocybe major (Nyl.) Körb.: 13 - Abies balsamea, 8079. S2S3, G4; rare (Wong and Brodo 1992).

$\uparrow$ Stenocybe pullatula (Ach.) Stein: $\mathbf{2}$ - Alnus incana ssp. rugosa, 8081.8 - Alnus incana ssp. rugosa, 8099. 9 - Alnus incana ssp. rugosa, 8065, 8097. 13 - Alnus incana ssp. rugosa, 8089, 8100.16 - Alnus incana ssp. rugosa, 8063. SU, GNR; rare (Wong and Brodo 1992).

Stereocaulon saxatile H. Magn.: 12 - granitic rock, 8685. S5, G5; common (Wong and Brodo 1992).

Stereocaulon tomentosum Fr.: 12 - soil, 8686. S4S5, G5; rare (Wong and Brodo 1992).

Trapelia placodioides Coppins \& P. James: 12 - rock, 9108 (NY). S5, GNR; common (Wong and Brodo 1992).

Trapeliopsis flexuosa (Fr.) Coppins \& P. James: 13 lignicolous stump, 8687. S4S5, G5; frequent (Wong and Brodo 1992).

Trapeliopsis granulosa (Hoffm.) Lumbsch: 12 - sandy soil, 8688. S5, GNR; common (Wong and Brodo 1992).
Usnea hirta (L.) F.H. Wigg.: 11 - Pinus resinosa, 8946. 12 - Rhus typhina, 8756. S4S5, G3G5; frequent (Wong and Brodo 1992).

Usnea subfloridana Stirt.: 9 - deciduous snag, 8974. 12 - snag, 8999. S4S5, GNR; rare (Wong and Brodo 1992).

Variolaria amara Ach.: 2 - snag, 8649. 8 - Thuja occidentalis, 9253.9 - snag, 9221. 20 - Thuja occidentalis, 8650. S4S5, G5?; frequent (Wong and Brodo 1992).

Verrucaria sp.: 12 - rock, 9107 (NY, OAC).

Violella fucata (Stirt.) T. Sprib. 2 - snag, 9152 (NY). SNR, GNR; not reported by Wong and Brodo (1992).

Vulpicida pinastri (Scop.) J.-E. Mattsson \& M.J. Lai: 12 - Rhus typhina snag, 8689. S4S5, G4G5; rare (Wong and Brodo 1992).

Xanthomendoza fallax (Hepp ex Arnold) Søchting, Kärnefelt \& S. Kondr.: 1 - Quercus rubra, 8696, 8697. S5, G5; very common (Wong and Brodo 1992).

Xanthomendoza ulophyllodes (Räsänen) Søchting, Kärnefelt \& S. Kondr.: 8 - Abies balsamea, 8791. SNR, GNR; not reported by Wong and Brodo (1992).

*Xanthoparmelia angustiphylla (Gyelnik) Hale: 9 rock, 9000. S1, G5; not reported by Wong and Brodo (1992).

Xanthoparmelia cumberlandia (Gyeln.) Hale: 9 - siliceous rock, 8691.10 - siliceous rock, 8692 . 12 - siliceous rock, 8693-8695, 8761. 16 siliceous rock, 8773. S5, G5; very common (Wong and Brodo 1992).

Xanthoparmelia viriduloumbrina (Gyelnik) Lendemer: 12 - rock, 8698. SNA, G5; common (Wong and Brodo 1992).

Xanthoria polycarpa (Hoffm.) Th. Fr. ex Rieber: 9 Picea glauca, 8760; Populus balsamifera, 8785. S4, G4G5; frequent (Wong and Brodo 1992).

\section{Discussion}

The Copeland Forest Resources Management Area is a refuge for lichen diversity in southern Ontario. The forest contains 129 (28\%) of the 465 species reported from the region by Wong and Brodo (1992), as well as an additional 25 species that were not on that list. This is despite the fact that the study area hosts only a small percentage of the variety of ecosystems that occur across the southern part of the province.

Two other extensive lichen diversity studies have been completed in specific regions of southern Ontario. The first study was in the Ottawa region, where 277 species were found on the Ontario side of the Ontario/ Quebec border (Brodo 1988). The second study was at Bruce Peninsula National Park of Canada and Fathom Five National Marine Park of Canada, where the 17th Tuckerman Workshop was held in 2008. A team of lichenologists at the workshop reported 365 species 
of lichens and allied fungi (Brodo et al., in press). The lichen richness in both of these studies is higher than in the Copeland Forest, but both areas have a greater number of ecosystems, which can be colonized by a greater number of species. The lichen richness found in these studies and in the Copeland Forest are not comparable because of the ecological differences and the substantially different degrees of disturbance.

Wong and Brodo (1992) found that 51 lichen species previously recorded from the province were no longer present. They hypothesized that this was due to a decline in air quality, an increase in development, and a loss of habitat. The Copeland Forest exemplifies this apparent decline in species richness, as no species typically used as indicators of good air quality were found (Henderson 2000; Cameron et al. 2007; McMullin and Ure 2008). Particular species that were notably absent in the Copeland Forest were those that contain cyanobacteria (cyanolichens). No cyanolichens were present, with the exception of the genus Peltigera, which is common even in disturbed areas.

The Copeland Forest Resources Management Area is a relatively large continuous woodland in southern Ontario. Many of the forests that remain in southern Ontario, particularly those in the southwestern part of the province, are fragmented. Fragmentation has been shown to reduce the diversity in species communities (Fahrig 2003). One of the reasons for this reduction is an increase in edge effects, which include an increase in light and wind exposure as well as a reduction in moisture compared with the forest interior (Chen et al. 1993; Renhorn et al. 1997; Esseen and Renhorn 1998). The Copeland Forest is large enough (1780 ha) to contain a significant forest interior, which includes many microhabitats for lichens to colonize.

Old-growth forests have been shown to have a greater lichen diversity because they contain a greater number of microhabitats than second-growth forests of the same area (Lesica et al. 1991; McMullin et al. 2008), but Lõhmus and Lõhmus (2011) recently showed that managed second-growth forests that cover a large area can also contain a wide variety of microhabitats and, therefore, a wide variety of species. The overall lichen diversity of the study area exemplifies the findings of Lõhmus and Lõhmus (2011). In other words, its large area contains a number of different microhabitats that support a wide variety of lichen communities, despite heavy recreational use and recent silvicultural disturbance.

Within the context of the study area, the alpha diversity in each of the 24 sites examined also differed considerably. The 6 sites with the highest lichen richness also had unique assemblages of species which helped to increase the overall number of species in the study area. Site 2 had the highest number of species (42). This site is in a protected river valley with a variety of mature trees along the valley slopes and exposed trees and snags in the floodplain of the Coldwater Riv- er. Moisture was high and sustained at this site, and the trees appeared to be older than most in the study area; this may be an area that was not harvested in the past.

Site 12 had the second highest number of species (40). This site contains an exposed area of rolling hills covered in sandy soil; it also contains the highest lichen biomass in the Copeland Forest, as the ground cover is dominated by species in the genus Cladonia subgenus Cladina. This exposed area is sheltered by the surrounding forest and contains exposed calcareous rocks, which are rare in the study area, where most rocks are siliceous.

Two similar sites with high diversity were 16 (40 species) and 8 (37 species). Both of these sites were large tracts of uninterrupted mixed hardwood forests with water bodies in close proximity, which presumbably maintain high and sustained levels of moisture. Site 9 (38 species) was along the same river that runs though site 2 . Where the Coldwater River runs through site 9 , however, it is not in a sheltered area and there is no floodplain with snags or trees. The microhabitats at this site include a variety of exposed trees, exposed rocks, and exposed cement on a river culvert. Site 13 (33 species) is a treed wetland with high light exposure and high levels of moisture that are sustained.

These 6 sites were the only ones of the 24 that contained more than 30 species each. Common variables among these sites included high light exposure and a variety of tree types, and moisture was generally higher. Many of the other sites have closed canopies, which may have limited the number of species able to colonize (Coxson and Coyle 2003; Coxson and Stevenson 2007).

These results are consistent with a recent study by McMullin et al. (2010), which showed that variation in light (canopy closure), moisture, soil, and tree types and ages were the primary environmental variables driving lichen diversity in forest ecosystems. Because of the variation in these sites, the lichen community structure in them differed. When combined, they increased the gamma diversity in the Copeland to a number much higher than any one site contained. Once again, these results support the species-area correlation described by Lõhmus and Lõhmus (2011).

This study contributes to a growing body of knowledge of the lichen biota in southern Ontario. The number of species now known from the southern region of the province has increased substantially since the account of Wong and Brodo (1992) (Matthes et al. 2000; Selva 2005; Brodo et al., in preparation; McMullin and Lewis, in preparation; Lewis et al., in preparation). Based on this study, provincial status ranks, the status given by Wong and Brodo (1992), the results from the lichen study on the Bruce Peninsula (Brodo et al, in preparation), and unpublished collections from southern Ontario (in the Lichenology Section of the National Herbarium of Canada, Canadian 
Museum of Nature, Ottawa, Ontario (CANL), in the Botany Department Herbarium, University of Guelph, Guelph, Ontario (OAC), and NY), the following lichen species are identified as species of interest in the Copeland Forest Resource Management Area: Anisomeridium polypori, Arthonia byssacea, A. ruana, Bellemerea cinereorufescens, Calicium trabinellum, Candelariella lutella, Chaenothecopsis debilis, Chaenothecopsis exilis, Chaenothecopsis pusiola, Cladonia digitata, Cladonia parasitica, Cresponea chloroconia, Lecidea sarcogynoides, Micarea micrococca, Multiclavula mucida, Pachyphiale fagicola, Parmeliopsis hyperopta, Phaeocalicium populneum, Phlyctis speirea, Placynthiella uliginosa, Porpidia cinereoatra, Stereocaulon tomentosum, Vulpicida pinastri, and Xanthoparmelia angustiphylla. These species appear to be uncommon in Canada, Ontario, or southern Ontario. Pachyphiale fagiolia is the only species reported that seems to be rare throughout its known range (Vězda and Poelt 1975; Ekman 1996). It is, however, a small and inconspicuous species that may be overlooked. Lecidea sarcogynoides is another seemingly rare species in North America. It is known from only one other unreported collection in North America, from Pennsylvania in 2008 (Lendemer 12598, NY), but it may have been overlooked in the past as well.

Micarea micrococca is collected and reported for the first time from Ontario, but it was only recently separated from M. prasina s.l. based on chemistry (Czarnota 2007; Czarnota and Guzow-Krzemińska 2010). This somewhat inconspicuous species is known from three neighbouring states in the United States, Michigan (Common 3344, at the Plant Biology Laboratories Herbarium, Michigan State University, East Lansing, Michigan (MSC)), New York (Common 3990, MSC), and Pennsylvania (Lendemer 16665, NY), so it is not surprising that it was found in Ontario.

Micarea micrococca is also reported for the first time in Canada, but three previous collections were made in 2008 in Newfoundland, Newfoundland and Labrador (Buck 944316, 944317, NY, and Lendemer 10093, NY).

Three additional species are reported for the first time in Ontario, but have been previously collected in the province-Bellemerea cinereorufescens, Phlyctis speirea, and Xanthoparmelia angustiphylla. Bellemerea cinereorufescens is collected for the third time in Ontario. It is typically an arctic and alpine species, so this range extension into southern Ontario is surprising (Figure 2C) (Thomson 1997). It was first collected in the province from the Thunder Bay Distinct in 1968 (Brodo 19705, CANL) and again in 1991 in Renfrew County in eastern Ontario (Brodo 27914, CANL). Phlyctis speirea was first collected from southern Ontario in 2010 (Lewis 392, CANL). Xanthoparmelia angustiphylla is known from New England and Minnesota (Wetmore 2005; Hinds and Hinds 2007), so it is not surprising that it was found in Ontario. The first collection of $X$. angustiphylla in the province is from 1983 in northern Ontario (Fischlin 160, CANL). Two other seemlingly rare species in Ontario that were collected in the Copeland Forest are Candelariella lutella and Chaenothecopsis exilis. Candelariella lutella is reported and collected for the second time in Ontario and the third time in Canada (Thomson 1997). Chaenothecopsis exilis was previously collected and reported from northeastern Ontario (McMullin 2011) and from the north shore of Lake Superior (Tibell 5389, Botany Section, Museum of Evolution, Uppsala University, Uppsala, Sweden (UPS)) (Selva and Tibell 1999).

Ten species with a provincial status rank of S1 (imperilled), S2 (imperilled), or S3 (vulnerable) were found in the Copeland Forest. Due to the lack of baseline data on the lichen biota in the province in the past, some of these ranks may no longer be accurate because of additional recent collections. Based on the results of the present study and recent collections made throughout Ontario, we recommend that the following species be reassessed and moved to a higher provincial rank: Chaenothecopsis pusiola, Cresponea chloroconia, Placynthiella uliginosa, Anisomeridium polypori, Chaenothecopsis debilis, Porpidia cinereoatra, Stenocybe major.

\section{Conclusion}

Lichen richness in the Copeland Forest Resources Management Area is high, and the site serves as a refuge for lichen diversity in southern Ontario, where lichen richness has been decreasing for more than a century (Wong and Brodo 1992). Our study has shown not only that this forest has potential conservation significance, but also that second-growth forests in general can support a diverse lichen biota in the province. While our results cannot a priori be extrapolated to secondary forests as a whole, they do indicate that large tracts of such forests merit study and consideration for the conservation and management of lichen biodiversity.

Future management plans for the Copeland Forest should include the preservation of lichen diversity and should target the sites with the greatest richness (sites $2,8,9,12,13$, and 16). Such plans should also specifically target uncommon species. If a single site is to be selected for protection in the Copeland Forest, site 12 is recommended. This site has a unique lichen community that includes many of the species of interest from this study and the only species that is new to Canada, Lecidea sarcogynoides. Site 1 is also recommended because it contains Pachyphiale fagicola, one of the only species found in the Copeland Forest that appears to be rare throughout its range.

The results from our study contribute to a better understanding of the understudied lichen biota of southern Ontario, particularly in second-growth forests. Most intensive lichen studies in the region have been in protected areas, such as national and provincial parks, 
in older and less disturbed forests (Matthes et al. 2000; Selva 2005; Brodo et al., in press; McMullin and Lewis, in press; Lewis et al., in preparation). These older forests are uncommon in southern Ontario, and younger second-growth forests that are intensively used now dominate the natural landscape.

Here we have shown that second-growth forests can be important refugia for lichen diversity. We found that sites with a high variation in canopy closure, tree species and age (particularly different deciduous and conifer trees), moisture, and the presence of snags have the highest lichen diversity. Forest managers in southern Ontario interested in preserving lichen diversity can use our results to help identify and protect areas of interest on their properties.

\section{Acknowledgements}

We gratefully acknowledge the following for their assistance with this project: Nancy Ironside and the Couchiching Conservancy for supporting our study; Ingrid van der Marel for hosting and being a guide in the Copeland Forest; Irwin Brodo and Stephen Clayden for helpful comments on the manuscript; Gloria McMullin for data entry; Dorthea Hangaard for logistic support throughout the project; Franziska BoerlinPetzold for curating specimens; Phil Gormaly and the Ontario Ministry of Natural Resources for producing field maps and the map in this report; Jessica Allen for images of Lecidea sarcogynoides; Irwin Brodo and Jose Maloles for identification assistance; Gord Michener, Nancy Ironside, and Ingrid van der Marel for assisting in the field; and Steven Newmaster and the Biodiversity Institute of Ontario for in-kind support.

\section{Literature Cited}

Ahti, T. 1964. Macrolichens and their zonal distribution in boreal and arctic Ontario, Canada. Annales Botanici Fennici 1: $1-35$.

Bates, D. V., and R. Sizto. 1987. Air pollution and hospital admissions in southern Ontario: the acid summer haze effect. Environmental Research. 43: 317-331.

Botting, R. S., and C. Delong. 2009. Macrolichen and bryophyte responses to coarse woody debris characteristics in sub-boreal spruce forest. Forest Ecology and Management 258: S85-S94.

Brodo, I. M. 1988. Lichens of the Ottawa Region, Second edition. Ottawa Field-Naturalists' Club Special Publication No. 3. Ottawa Field-Naturalists' Club, Ottawa, Ontario. 115 pages.

Brodo, I. M., S. D. Sharnoff, and S. Sharnoff. 2001. Lichens of North America. Yale University Press, New Haven, Connecticutt. 795 pages.

Brummitt, R. K., and C. E. Powell. Editors. 1996. Authors of Plant Names. Royal Botanical Gardens, Kew, U.K. 732 pages.

Cameron, R. P., T. Neily, and D. H. S. Richardson. 2007. Macrolichen indicators of air quality for Nova Scotia. Northeastern Naturalist 14: 1-14.

Chen, J., J. F. Franklin, and T. A. Spies. 1993. Contrasting microclimates among clearcut, edge and interior of old- growth Douglas-fir forest. Agricultural and Forest Meteorology 63: 219-237.

Coxson, D. S., and M. Coyle. 2003. Niche partitioning and photosynthetic response of alectorioid lichens from subalpine spruce-fir forest in north-central British Columbia, Canada: the role of canopy microclimate gradients. Lichenologist 35: 157-175.

Coxson, D. S., and S. K. Stevenson. 2007. Growth rate responses of Lobaria pulmonaria to canopy structure in even-aged and old-growth cedar-hemlock forests of central-interior British Columbia, Canada. Forest Ecology and Management 242: 5-16.

Culberson, C. F., and H. Kristinsson. 1970. A standardized method for the identification of lichen products. Journal of Chromatography 46: 85-93.

Czarnota, P. 2007. The lichen genus Micarea (Lecanorales, Ascomycota) in Poland. Polish Botanical Studies 23: 1199.

Czarnota, P., and B. Guzow-Krzemińska. 2010. A phylogenetic study of the Micarea prasina group shows that Micarea micrococca includes three distinct lineages. Lichenologist 42: 7-21.

Ekman, S. 1996. The corticolous and lignicolous species of Bacidia and Bacidina in North America. Opera Botanica 127: 1-148.

Environment Canada. 2012. National Climate Data and Information Archive. http://climate.weatheroffice.gc.ca. (Accessed March 3, 2012).

Esseen, P., and K. Renhorn. 1998. Edge effects on an epiphytic lichen in fragmented forests. Conservation Biology 12: 1307-1317.

Esslinger, T. L. 2012. A cumulative checklist for the lichenforming, lichenicolous and allied fungi of the continental United States and Canada. North Dakota State University, Fargo, North Dakota. (First posted 1 December 1997; most recent version (\#18) 13 December 2012). http://www.ndsu .edu/pubweb/ esslinge/chcklst/chcklst7.htm.

Fahrig, L. 2003. Effects of habitat fragmentation on biodiversity. Annual Review of Ecology, Evolution and Systematics 34: 487-515.

Golas, I. 1980. The Copeland Forest Resources Management Area: A History - 1800-1978. Reported prepared for the Ontario Ministry of Natural Resources, Midhurst, Ontario. 61 pages.

Gowan, S. P., and I. M. Brodo. 1988. The lichens of Fundy National Park, New Brunswick, Canada. Bryologist 91: 255-325.

Goward, T. 1994. Notes on oldgrowth-dependent epiphytic macrolichens in inland British Columbia, Canada. Acta Botanica Fennica 150: 31-38.

Henderson, A. 2000. Literature on air pollution and lichens XLIX. Lichenologist 32: 89-102.

Henry, M., and P. Quinby. 2010. Ontario's Old-Growth Forests. A Guidebook Complete with History, Ecology and Maps. Fitzhenry and Whiteside Limited, Markham, Ontario. 232 pages.

Hinds, J. W., and P. L. Hinds. 2007. The Macrolichens of New England. New York Botanical Garden Press, Bronx, New York. 584 pages.

Kenkel, N. C., and G. E. Bradfield. 1986. Epiphytic vegetation on Acer macrophyllum: a multivariate study of species-habitat relationships. Vegetation 68: 43-53. 
Kuusinen, M. 1996. Epiphytic flora and diversity on basal trunks of six old-growth forest tree species in southern and middle boreal Finland. Lichenologist 28: 443-463.

Lesica, P., B. McCune, S. V. Cooper, and W. S. Hong. 1991. Differences in lichen and bryophyte communities between old-growth and managed second-growth forests in the Swan Valley, Montana. Canadian Journal of Botany 69: $1745-1755$

Lõhmus, A., and P. Lõhmus. 2011. Old-forest species: the importance of specific substrata vs. stand continuity in the case of calicioid fungi. Silva Fennica 45: 1015-1039.

Matthes, U., B. D., Ryan, and D. W. Larson. 2000. Community structure of epilithic lichens on the cliffs of the Niagara Escarpment, Ontario, Canada. Plant Ecology 148: 233-244.

McMullin, R. T. 2011. Relationships between silviculture, lichen diversity and woodland caribou (Rangifer tarandus caribou) in northern Ontario. Ph.D. thesis, University of Guelph, Guelph, Ontario. 553 pages.

McMullin, R. T., and D. Ure. 2008. Lichen monitoring protocol for Kejimkujik National Park and National Historic Site of Canada. Report produced for Parks Canada, Maitland Bridge, Nova Scotia. 43 pages.

McMullin, R. T., P. N. Duinker, R. P. Cameron, D. H. S. Richardson, and I. M. Brodo. 2008. Lichens of coniferous old-growth forests of southwestern Nova Scotia, Canada: diversity and present status. Bryologist 111: 620-637.

McMullin, R. T., P. N. Duinker, D. H. S. Richardson, R. P. Cameron, D. C. Hamilton, and S. G. Newmaster. 2010. Relationships between the structural complexity and lichen community in coniferous forests of southwestern Nova Scotia. Forest Ecology and Management 260: 744 749.

McMullin, R. T., I. D. Thompson, B. W. Lacey, and S. G. Newmaster. 2011. Estimating the biomass of woodland caribou forage lichens. Canadian Journal of Forest Research 41: 1961-1969.

Nash, T. H., III. 2008. Lichen Biology. Second edition. Cambridge University Press, New York, New York. 486 pages.

Newmaster, S. G., R. J. Belland, A. Arsenault, D. H. Vitt, and T. R. Stephens. 2005. The ones we left behind: comparing plot sampling and floristic habitat sampling for estimating bryophyte diversity. Diversity and Distributions 11 : $57-72$.

Ontario Ministry of Natural Resources. 2012. Ontario's Species at Risk Program. http://www.mnr.gov.on.ca/en /Business/Species/index.html. (Accessed March 31, 2012).

Orange, A., P. W. James, and F. J. White. 2001. Microchemical Methods for the Identification of Lichens. British Lichen Society, London, U.K. 101 pages.
Perera A. H., D. L. Euler, and I. D. Thompson. Editors. 2001. Ecology of a Managed Terrestrial Landscape: Patterns and Processes of Forest Landscapes in Ontario. UBC Press, Vancouver, British Columbia. 336 pages.

Renhorn, K. E., P. A. Esseen, K. Palmqvist, and B. Sundberg. 1997. Growth and vitality of epiphytic lichens I. Responses to microclimate along a forest edge-interior gradient. Oecologia 109: 1-9.

Rowe, J. S. 1972. Forest Regions of Canada. Department of the Environment, Canadian Forestry Service. Publication No. 1300.172 pages.

Schmitt, C. K., and N. G. Slack. 1990. Host specificity of epiphytic lichens and bryophytes: a comparison of the Adirondack Mountains (New York) and the Southern Blue Ridge Mountains (North Carolina). Bryologist 93: 257274.

Selva, S. B. 1999. Survey of epiphytic lichens of late successional northern hardwoods forests in northern Cape Breton Island. Cape Breton Highlands National Park and Parks Canada, Ingonish Beach, Nova Scotia.

Selva, S. B. 2003. Using calicioid lichens and fungi to assess ecological continuity in the Acadian Forest ecoregion of the Canadian Maritimes. Forestry Chronicle 79: 550-558.

Selva, S. B. 2005. Using calicioid lichens and fungi to assess ecological continuity of four forest stands in the White Lakes region of southern Ontario. A report prepared for the Ontario Ministry of Natural Resources, Kemptville, Ontario. 18 pages.

Selva, S. B., and L. Tibell. 1999. Lichenized and non-lichenized calicioid fungi from North America. Bryologist 102: $377-397$.

Söderström, L. 1988. Sequence of bryophytes and lichens in relation to substrate variables of decaying coniferous wood in northern Sweden. Nordic Journal of Botany 8: 89-97.

Thomson, J. W. 1997. American Arctic Lichens 2. The Microlichens. University of Wisconsin Press, Madison, Wisconsin. 675 pages.

Vězda, A., and J. Poelt. 1975. Die Gattungen Dimerella und Pachyphiale. Pages 127-132 in Khumbu Himal; Ergebnisse des Froschungsunternehmens Nepal Himalaya. Edited by W. Hellmich. Band 6/2. Springer-Verlag, Berlin.

Wetmore, C. 2005. Keys to the Lichens of Minnesota. University of Minnesota, St. Paul, Minnesota. 92 pages.

Wong, P. Y., and I. M. Brodo. 1992. The lichens of southern Ontario. Syllogeus 69: 1-79.

Received: 12 April 2013

Accepted 7 May 2013. 